\title{
Linfoma de amígdala: reporte de un caso pediátrico
} Palatine tonsil lymphoma: a pediatric case report

\author{
Dra. Roxana Spini ${ }^{a}$ Dr. Daniel Cruz ${ }^{a}$, Dra. Lucía Fernández y Dra Mariana Juchlia
}

\section{RESUMEN}

El linfoma es la neoplasia maligna de cabeza y cuello más común en la población pediátrica. Las manifestaciones clínicas de linfoma en amígdala palatina son generalmente asimetría amigdalina, alteración en la apariencia de la mucosa y adenopatías cervicales.

Ante una hipertrofia amigdalina unilateral, se debe diferenciar de cuadros infecciosos, procesos crónicos, otros tumores o simplemente una hiperplasia amigdalina benigna. El seguimiento estricto de la asimetría amigdalina es un pilar importante a la hora del diagnóstico de los pacientes con linfoma amigdalino, debido a que un tratamiento temprano es fundamental para el pronóstico.

Se presenta una niña de 14 años con hipertrofia amigdalina unilateral y odinofagia, de aproximadamente 21 días de evolución sin respuesta al tratamiento antibiótico, con diagnóstico de linfoma de Burkitt. Presenta remisión completa de la enfermedad al año del diagnóstico.

Palabras clave: linfoma, niño, neoplasias tonsilares, linfoma de Burkitt.

\section{ABSTRACT}

Lymphoma is the most common childhood malignancy in the head and neck. The most common clinical manifestations of lymphoma in palatine tonsil are the tonsils asymmetry, alteration in the appearance of the mucous and cervical lymphadenopathy.

The unilateral tonsillar hypertrophy must be differentiated with infectious processes, with chronic processes, other tumors or benign tonsillar hyperplasia. The monitoring of tonsillar hypertrophy is strict, because early diagnosis and treatment are of great importance in the prognosis of tonsillar lymphoma patients.

A 14 year-old girl presents unilateral tonsillar enlargement and odynophagia. After 21 days of antibiotic treatment, she has not clinical improvement. The diagnosis is Burkitt's lymphoma. One year after diagnosis, she presents complete remission of the disease.

Keywords: lymphoma, child, tonsillar neoplasms, Burkitt's lymphoma.

a. División de Otorrinolaringología.

b. Jefa de residentes de Otorrinolaringología Infantil.

Hospital General de Niños Pedro de Elizalde,

Ciudad Autónoma de Buenos Aires, Argentina.

Correspondencia:

Dra. Roxana Spini: roxanaspini@intramed.net

Financiamiento: Ninguno.

Conflicto de intereses: Ninguno que declarar.

Recibido: 5-8-2020

Aceptado: 10-2-2021 http: / / dx.doi.org/10.5546/ aap.2021.e330

Cómo citar: Spini R, Cruz D, Fernández L, Juchli M. Linfoma de amígdala: reporte de un caso pediátrico. Arch Argent Pediatr 2021;119(4):e330-e334.

\section{INTRODUCCIÓN}

El linfoma es el tumor maligno infantil más común en la cabeza y el cuello. En el $15 \%$ de los casos, afecta al anillo de Waldeyer. ${ }^{1}$

El linfoma es la tercera neoplasia en pediatría, siendo más frecuente el linfoma no Hodgkin (LNH). El LNH consiste en una adenopatía cervical indurada y crónica, aunque el 4-20\% se presenta como manifestación extraganglionar en cabeza y cuello, especialmente en las amígdalas palatinas. $^{2}$

La localización extraganglionar incluye el compromiso amigdalino, nasofaríngeo y de base de lengua. ${ }^{3}$

Se presenta una niña de 14 años que recibió tratamiento por un absceso periamigdalino, pero sin respuesta, con diagnóstico posterior de linfoma de Burkitt (LB).

\section{CASO CLÍNICO}

Niña de 14 años que consulta a la guardia de nuestro hospital por presentar odinofagia persistente a pesar del tratamiento con amoxicilina $90 \mathrm{mg} / \mathrm{kg} /$ día una semana previa, con diagnóstico presuntivo de faringitis. Refiere que no se le realizó hisopado. Al examen físico presenta hipertrofia amigdalina (HA) derecha con desplazamiento hacia la línea media y abombamiento del paladar blando. Por la evolución tórpida y sospecha de absceso periamigdalino derecho, se decide internar a la paciente para tratamiento parenteral con ceftriaxona (50 mg/ kg/día) y clindamicina (30 mg/kg/día).

Se realiza interconsulta a Otorrinolaringología: Paciente en regular estado general, dolorida, afebril. Tumoración de amígdala derecha, desplazamiento hacia la línea media, marcado abombamiento del paladar blando homolateral con áreas de ulceración en su cara medial, de 
FIGURA 1. La paciente bajo anestesia general e intubada en quirófano. Se puede observar la hipertrofia amigdalina derecha (flecha blanca) con áreas de ulceración en el paladar blando (flecha negra)

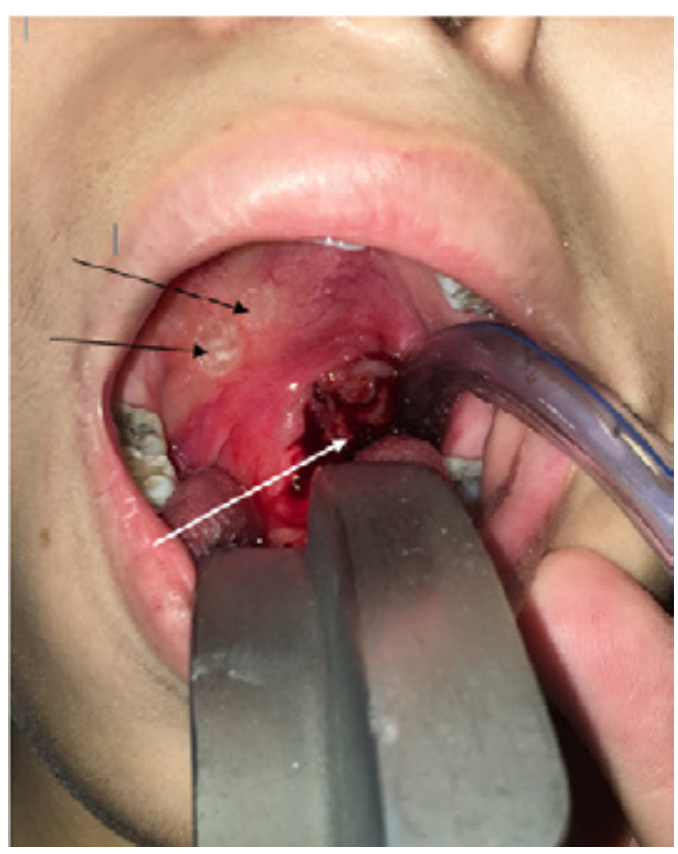

consistencia dura a la palpación (Figura 1). Amígdala izquierda normal grado II de Brodsky. ${ }^{4}$ Adenopatías cervicales ipsilaterales indoloras. $\mathrm{Al}$ interrogatorio, la paciente niega trismus durante toda su evolución. Presenta dificultad progresiva para la alimentación, para el habla y aparición de ronquido.

Se solicita tomografía computada (TC) de macizo cráneo facial y de cuello con contraste, y radiografía de tórax frente y perfil. Hematooncología solicita laboratorio completo por sospecha de lisis tumoral.

La radiografía de tórax fue normal. Laboratorio: glóbulos blancos (gb): $9090 / \mathrm{mm}^{3}$ (neutrófilos $49 \%$ ); hemoglobina 14,2 g/ dl; hematocrito 44,8\%; plaquetas $315000 / \mathrm{mm}^{3}$; eritrosedimentación $6 \mathrm{~mm} / \mathrm{h}$; glucemia $92 \mathrm{mg} / \mathrm{dl}$; función renal (urea y creatininemia), calcemia, fosfatemia, magnesemia, uricemia, hepatograma completo, lipidograma, proteínas totales y albumina, ionograma y coagulograma dentro de los valores normales.

La TC cervical evidencia imagen hipodensa que ocupa fosa amigdalina derecha y se extiende a rino-, oro- e hipofaringe, que comprime

FIGURA 2. Tomografía computada de macizo craneofacial y cuello con contraste. A-C: cortes axiales; D y E: cortes coronales; F: corte sagital. Se visualiza tumoración de amígdala (flecha negra): imagen hipodensa algo heterogénea que obstruye la luz de nasofaringe $y$ orofaringe
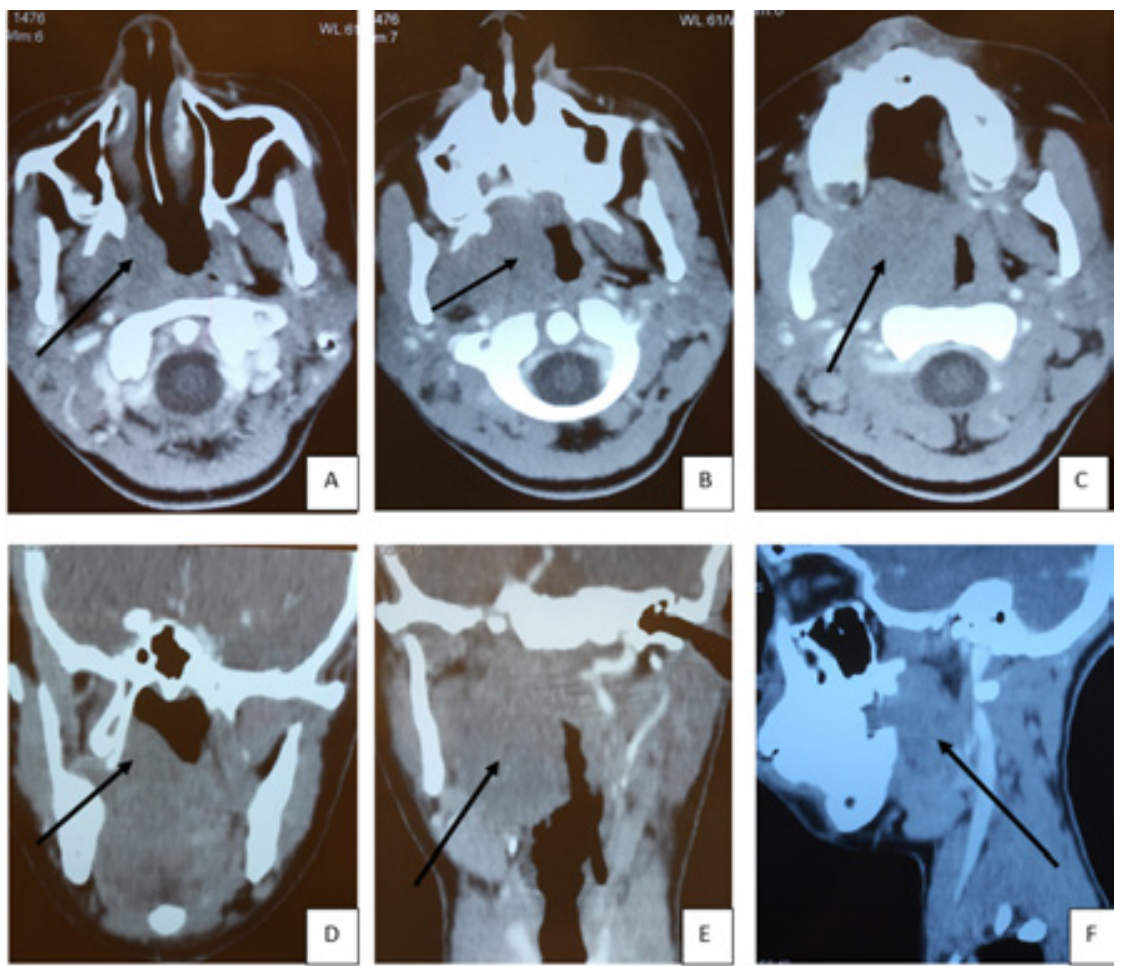
planos musculares profundos desplazando paquete vasculonervioso del cuello (Figura 2). Se solicita resonancia magnética $(\mathrm{RM})$ que informa formación hiperintensa en T2 e isointensa en
T1 que alcanza un diámetro de 57 x $37 \mathrm{~mm}$ colapsando totalmente la luz orofaríngea (Figura 3). Desplaza las estructuras adyacentes y el paquete vasculonervioso, pero sin invadirlo.

Figura 3. Resonancia magnética de macizo craneofacial y cuello con gadolinio. A: corte axial con gadolinio; B: corte sagital; C: corte coronal. Se observa una formación isointensa en T1 que alcanza un diámetro de $57 \times 37 \mathrm{~mm}$, que colapsa totalmente la luz orofaringea (flecha blanca), y desplaza las estructuras adyacentes y el paquete vasculonervioso, pero sin invadirlo
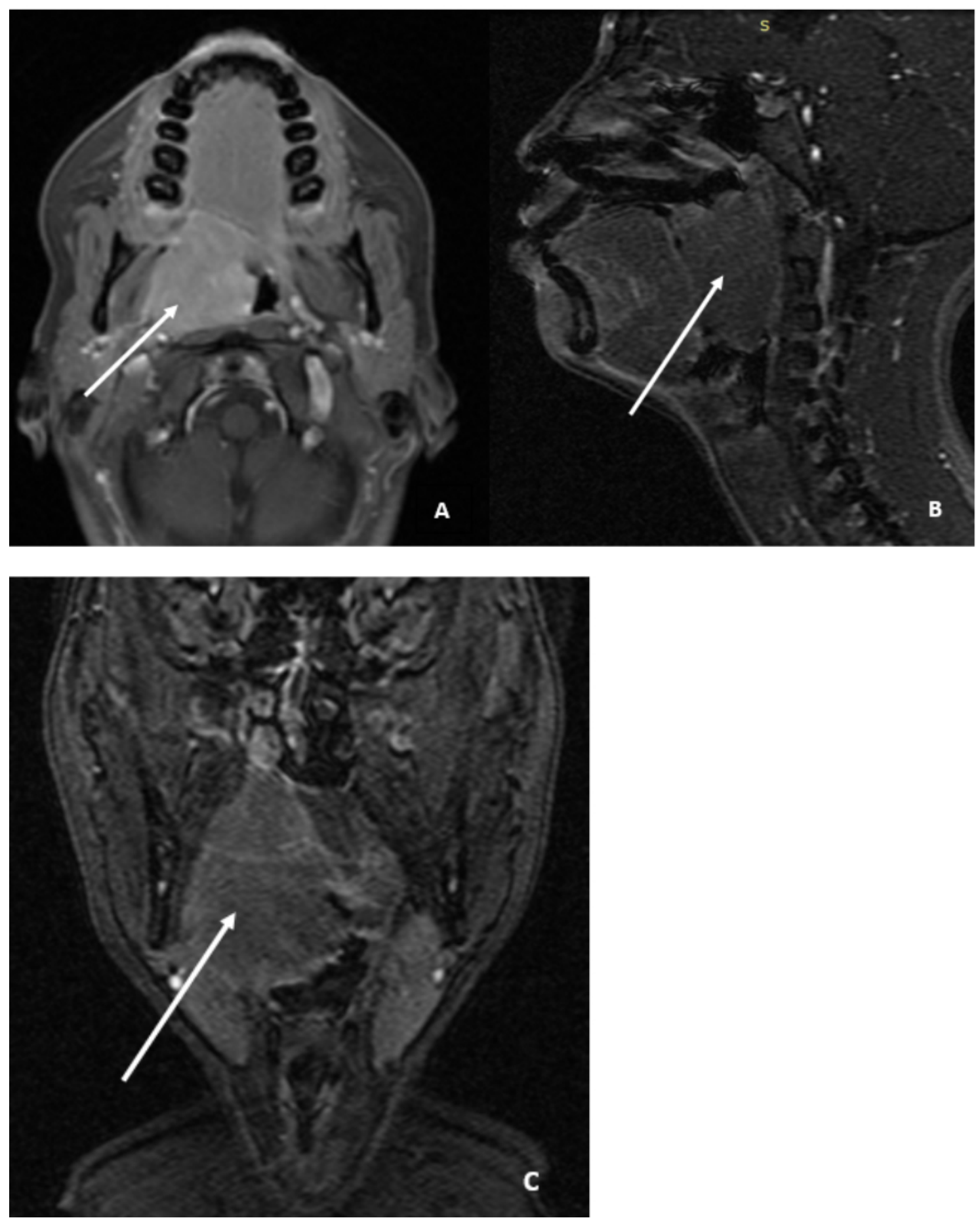
Por todo lo descripto, se decide efectuar toma de biopsia.

La biopsia se realiza por vía transoral, bajo anestesia general. Se extrae tejido amigdalino con bisturí frío (Figura 4). La anatomía patológica informa proliferación difusa de células linfoides atípicas. La inmunohistoquímica revela Bc16 positivo heterogéneo (60\% de las células B) y Ki67 positivo (100\% de las células B). Se confirma el diagnóstico de LNH de células B maduras de alto grado (LB).

Se solicita TC corporal, punción lumbar, punción aspiración de médula ósea y centellograma óseo, donde se evidencia la ausencia de lesiones metastásicas. Los estudios serológicos para el virus de Epstein Barr (VEB) fueron negativos y las inmunoglobulinas séricas, normales. Se presume linfoma extranodal en estadio II (IIE de Ann Arbor) ${ }^{5}$ y se inicia tratamiento con quimioterapia combinada endovenosa, oral e intratecal según protocolo. La paciente presenta buena evolución clínica al mes (Figura 5), entrando en remisión a los 3 meses del diagnóstico.

\section{DISCUSIÓN}

El LNH en pediatría se presenta como linfoma B de células maduras (llamado LB) y linfoma $B$ de células difusas. El LB es más común en varones, es altamente agresivo y se caracteriza por presentar lisis tumoral y buena respuesta a la quimioterapia. ${ }^{5}$

El principal signo de alarma de una neoplasia es la constatación de asimetría amigdalina dada por una significativa HA unilateral.

Figura 4. Muestra de biopsia de amígdala con bisturífrío

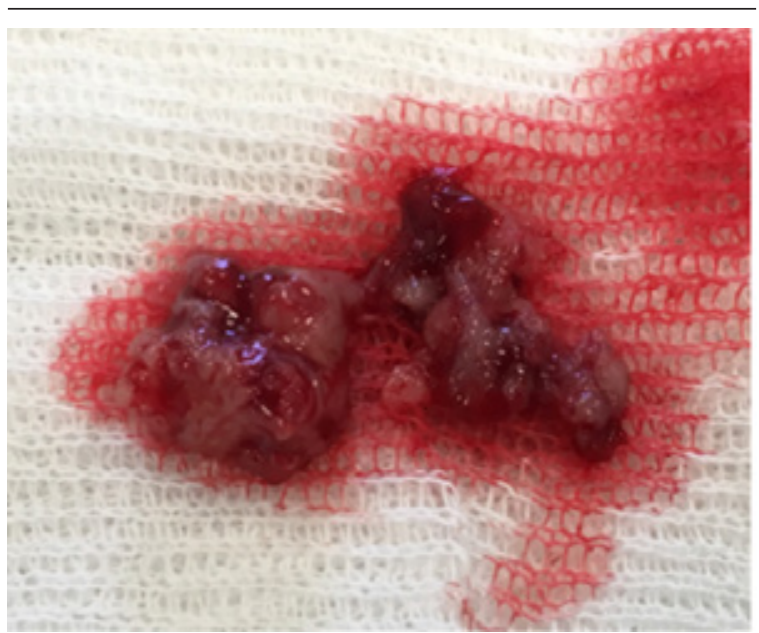

Sin embargo, la asimetría amigdalina es, con frecuencia, secundaria a una hiperplasia benigna o una falsa asimetría creada por la diferencia en la profundidad de la fosa tonsilar. ${ }^{1} \mathrm{Si}$ bien la incidencia de malignidad es baja (2,5 casos cada 10000 amigdalectomías), la asimetría amigdalina es uno de los hallazgos sugestivos de patología maligna. ${ }^{6}$

La mayoría de los pacientes con linfoma presentan un crecimiento amigdalino dentro de un período de 6 semanas previo al diagnóstico, adenopatía $>3 \mathrm{~cm}$, disfagia y la HA con cambios en la apariencia de la mucosa como ulceración o alteración de la coloración. ${ }^{1,7}$ En ocasiones puede ser confundida con infecciones, ya que las manifestaciones clínicas no son específicas, ocasionando el diagnóstico tardío de linfoma. ${ }^{2,8}$

También puede acompañarse de síntomas constitucionales, como pérdida de peso, fiebre y sudor nocturno. Se puede manifestar con odinofagia u otalgia ipsilateral, amigdalitis recurrente, roncopatía y apneas. Rara vez, es asintomático.

Los criterios de sospecha de malignidad son la aparición de HA unilateral, el aspecto

FIGURA 5. Hipertrofia amigdalina de grado I con resolución completa de la tumoración al mes del comienzo de la quimioterapia: se observan fauces libres

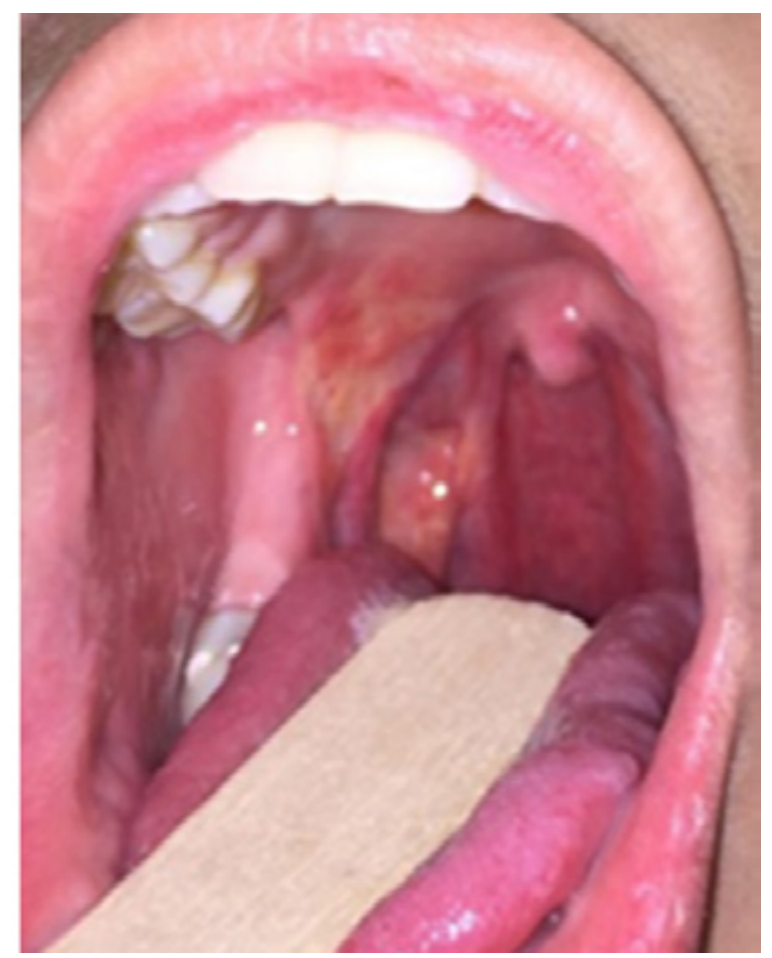


sospechoso de la amígdala palatina y las adenopatías. ${ }^{5}$ Otros diagnósticos diferenciales son infecciones crónicas (tuberculosis, actinomicosis), enfermedades granulomatosas (sarcoidosis) y tumores benignos (papilomas). ${ }^{5}$ La HA unilateral (asimetría amigdalina) sospechosa se produce en un período agudo, por lo general en un lapso menor a 6 semanas. El seguimiento estricto de la asimetría amigdalina es un pilar importante a la hora del diagnóstico de los pacientes con linfoma amigdalino, debido a que un tratamiento temprano es fundamental para el pronóstico.

El diagnóstico lo proporciona la anatomía patológica. El estudio inmunohistoquímico define la variedad celular. Idealmente la indicación es amigdalectomía (biopsia excisional) y analizar la amígdala en su totalidad. Sin embargo, en algunos casos los planos quirúrgicos están alterados o muy avanzados, alcanzan grandes tamaños, por lo cual es difícil realizar amigdalectomía en forma completa. En estos casos, es importante obtener una muestra amplia, dado que para los patólogos es un gran desafío diferenciar microscópicamente el LB, el linfoma linfoblástico, el linfoma blastoide de células del manto y el linfoma B de células maduras. $^{2}$

La TC es importante para delimitar la extensión tumoral, así como los estudios de líquido cefalorraquídeo y de médula ósea. En nuestro caso, se trató de un estadio II, que consiste en la infiltración localizada de un solo órgano o sitio extranodal con afectación ganglionar regional. ${ }^{7} \mathrm{La}$ RM es complementaria a la TC. Un estudio no reemplaza al otro. La RM identifica la extensión y los límites de diferentes tejidos blandos (como vasos sanguíneos, tejidos, adenopatías, etc.) siendo fundamental a la hora de realizar diagnóstico diferencial con otros tumores de cuello.

El tratamiento se basa en quimioterapia. ${ }^{5,8} \mathrm{Si}$ bien el LB es una neoplasia altamente agresiva, tiene buen pronóstico en estadios tempranos y en población pediátrica, siendo la tasa de supervivencia a los 5 años mayor al $90 \%$.,7

\section{Agradecimientos}

Los autores agradecen a la Dra. Elena Graciela por sus aportes y contribuciones en el diagnóstico de la paciente.

\section{REFERENCIAS}

1. Cuestas G, Martínez Font A, Demarchi M, Martínez Corvalan $\mathrm{M}$, et al. Linfoma de amígdala en niño con asimetría tonsilar. Caso clínico. Arch Argent Pediatr. 2015; 113(4):e219-22.

2. Toader C, Toader M, Stoica A, Pop G, et al. Tonsillar lymphoma masquerading as obstructive sleep apnea pediatric case report. Rom J Morphol Embryol. 2016; 57(2 Suppl):885-91.

3. Gaini RM, Romagnoli M, Sala A, Garavello W. Lymphomas of head and neck in pediatric patients. Int J Pediatr Otorhinolaryngol. 2009; 73 (Suppl 1):S65-70.

4. Brodsky L. Modern assessment of tonsils and adenoids. Pediatr Clin North Am. 1989; 36(6):1551-69.

5. Guimarães AC, de Carvalho GM, Bento LR, Correa C, Gusmão RJ. Clinical manifestations in children with tonsillar lymphoma: a systematic review. Crit Rev Oncol Hematol. 2014; 90(2):146-51.

6. Adil E, Medina G, Cunningham M. Pediatric Tonsil Cancer: A National and Institutional Perspective. J Pediatr. 2018; 197:255-61.

7. Sunkaraneni VS, Jones SE, Prasai A, Fish BM. Is unilateral tonsillar enlargement alone an indication for tonsillectomy? J Laryngol Otol. 2006; 120(7):E21.

8. Siupšinskienė N, Padervinskis E, Poškienė L, Endeley N, Vaitkus S. An unusual case of a sore throat and otalgia in a 4-year-old boy. Medicina (Kaunas). 2012; 48(5):277-9. 